\title{
Andy Pereira (ed): Plant Reverse Genetics: Methods and Protocols. Methods in Molecular Biology 678
}

\author{
The Humana Press, ISBN 978-1-60761-681-8
}

\author{
Stefano Meneghetti
}

(C) Springer Science+Business Media, LLC 2012

This book is an overview of the main functional genomics approaches and methods.

The book describes analysis of high-throughput genome sequence data, identification of non-coding RNA from sequence information, comprehensive analysis of gene expression by microarrays, mutational approaches to ascribe gene function by insertion sequences such as T-DNA and transposons for the silencing and overexpression of genes, metabolomic analysis, all of which supported by scripts to aid their computational use.

Even though any chapter introduces the basic knowledge of the treated topic, it is mainly aimed to provide detailed protocols describing how to start to work step by step, making this easy to read and understand.

Post-genomic tools from gene expression, proteomic and metabolomic techniques to gene silencing and phenomic are investigated, making it appealing and approachable for who starts at first time to work on it in the same way that people who want to keep informed on the latest trends.
In addition, I found particularly useful the effort to mention also software and database newly implemented to integrate lab tools.

I recommend the first chapter that gives an excellent account of the opportunities offered (revolution created) by next-generation sequencing technologies, underlying the applications and in particular highlighting the impellent need to manage and analyse the continuing increasing of sequencing outputs.

New program have to be developed to cope with the bottleneck created by short-reads sequences generated by NGS especially in re-sequencing application to find sequence polymorphisms.

A detailed description of the prerequisites, the requirements and the formats of the output data is presented, giving a useful list of alignment softwares.

Each chapter is followed by notes that are a very useful technical tips that are useful to students willing to learn how to reason when facing unforeseen occurrences.

Overall the book is well-conceived and useful. 